\title{
Tradição e mudança: os desafios da afirmação de marca na Andreato Comunicação e Cultura*
}

Juliana Winkel

Jornalista, especialista em Gestão da Comunicação, mestranda em Ciências da Comunicação e editora executiva da revista Comunicação \& Educação.

E-mail: juli.santos@usp.br

Resumo: O presente artigo traz uma reflexão a respeito da transição entre o modelo moderno de economia, política e produção cultural, predominante até meados do século $X X$, e o modelo pós-moderno, que a presente geração em idade produtiva vivencia e constrói. A análise se fecha, especialmente, sobre a trajetória do estúdio Andreato Comunicação e Cultura, à luz das transformações do mundo e do mercado nos últimos trinta anos - transformações essas que influem profundamente na cultura organizacional da empresa, assim como na maneira pela qual os profissionais da organização tratam os principais temas de seus produtos: a história e a cultura brasileiras. Por meio da análise da trajetória da organização - e especialmente de seu fundador, o artista gráfico Elifas Andreato - e de pesquisas mercadológicas e de relacionamento, este trabalho pretende indicar caminhos para fortalecer, perante o mercado, o vínculo da marca da empresa a temas ligados à cultura nacional. Ao mesmo tempo, enumera caminhos para que a organização possa melhor se expressar e, assim, dar continuidade à sua sólida história de produção cultural por meio do uso de novas mídias, em especial da Internet.

Palavras-chave: Comunicação, cultura, identidade, artes gráficas, trabalho, modernismo, pós-modernismo, Internet.
Abstract: The present study brings up a reflection about the transition between the modern model concerned to economy, politics and cultural production that prevailed up to the middle of the twentieth century, and the post-modern model, which the nowadays generation in productive age experiences and constructs. The analysis closes itself, specially, on the trajectory of Andreato Communication and Culture studio. Taking into account the world and market transformations in the latest thirty years - which influence intensively in the organizational culture of the enterprise, as well as in the way the professionals from the organization deal with the main themes related to their products: the Brazilian history and culture. Through the organization trajectory analysis - and specially the trajectory analysis of its founder, the graphic artist Elifas Andreato - and through market and relationship researches, this study intends to show ways to strengthen, in front of the market, the enterprise's brand vinculum to themes connected to the national culture. At the same time, it enumerates ways so that the organization can better express itself, and thus give continuity to its cultural production solid History by the use of new medias, in special Internet.

Keywords: Communication, culture, identity, graphic arts, work, modernism, postmodernism, Internet.

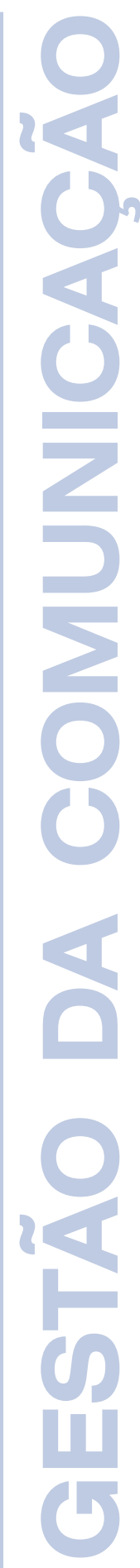

* Artigo escrito com base no trabalho de conclusão do curso Gestão da Comunicação, elaborado em dezembro de 2005. 
comunicação \& educação • Ano XVII • número 1 • jan/jun 2012

\section{INTRODUÇÃO}

Toda a reprodução que ultrapassa o imediato na vida cotidiana deixa de ser cotidiana. [...] Esta passagem ocorre, como diz Agnes Heller, quando se rompe com a cotidianidade; quando um projeto, uma obra ou um ideal convoca a inteireza de nossas forças e então suprime a heterogeneidade ${ }^{1}$.

Este artigo desenvolve-se a partir do trabalho realizado junto à Andreato Comunicação e Cultura, empresa voltada à produção de peças de comunicação ligadas à história e cultura brasileiras. $\mathrm{O}$ momento de crescimento da organização, durante o período de realização deste trabalho, coincidia com a necessidade de ampliação de seu posicionamento no terreno da comunicação - utilizando todo o capital artístico de sua equipe e de seu fundador, o artista gráfico Elifas Andreato, na busca de trabalhos mais abrangentes nas áreas cultural e empresarial, envolvendo especialmente a utilização de novas mídias.

Observando os canais de comunicação utilizados para alcançar seu público, as atenções do estudo foram voltadas às possíveis maneiras de aumentar a repercussão da mensagem institucional - com especial interesse pelo posicionamento digital da organização, área que oferece potencialidades muito aumentadas de comunicação e, ao mesmo tempo, pode traduzir com eficácia o perfil ali almejado: o de uma empresa fortemente identificada com a cultura nacional e disposta a acompanhar as tendências comerciais da pós-modernidade, ao mesmo tempo em que traz consigo a herança e o compromisso de atuação em momentos marcantes da história do país. Como objetivo principal da pesquisa, um desafio apontado com unanimidade pelos integrantes da organização: a busca, em meio a uma história já sólida de atuação, pela modernização de seu sistema de trabalho, aliando a rapidez da informação à busca por diferenciais num mercado altamente competitivo.

\section{DE OPERÁRIO A ARTISTA DE PROTESTO}

Na década de 1960, São Paulo vivia a efervescência cultural motivada pela prosperidade industrial que atingia a todos os centros urbanos. O governo de Juscelino Kubitschek estimulara a industrialização, fazendo aumentar as concentrações de trabalhadores urbanos, que se organizariam em sindicatos para exigir direitos trabalhistas².

Motivados pelo aumento da vida social e da renda, ganhavam vida gruCarmo, Cotidiano: nhecimento e crítica. São Paulo: Cortez, 1989. p. 25.

2. ARBEX JR, José. O Terceiro Mundo - Américas. s/l, s/d. Disponível em: <http://www.tvcultura. com.br/aloescola/historia/guerrafria/guerra11/ terceiromundo-americas. htm >. Acesso em: 16 ago. 2005.

pos e associações de diversos tipos: musicais, esportivos, educacionais. Novos conceitos nas artes e na cultura transpareciam nos palcos do Teatro de Arena, do Teatro Brasileiro de Comédia e do Teatro Oficina. Por meio de novidades estéticas e cênicas, essas companhias abordavam a situação do proletariado brasileiro, assim como os conflitos de classes que se anunciavam na nova sociedade industrial.

Elifas Andreato, que, ainda menino, migrara com a família do Paraná para São Paulo, na adolescência mostrou talento para o desenho, por meio das 
Tradição e mudança • Juliana Winkel

charges feitas ao jornal dos operários da indústria onde era torneiro mecânico. A opção pela carreira artística, mais tarde, coincidiria com a eclosão da ditadura militar e da censura. Elifas aprendeu o ofício de artista gráfico dentro da Editora Abril, um dos maiores grupos editoriais do país, ao mesmo tempo em que militava em publicações alternativas - atuação que abraçaria em tempo integral a partir da década de 1970.

No final da década de 1970, a demanda crescente por trabalhos fez surgir a necessidade de um local específico que centralizasse sua produção como artista gráfico. Em 1979, organizou um estúdio em São Paulo, ao lado de outros artistas gráficos. Durante duas décadas, o local funcionou como ponto de reunião para trabalhos individuais ou em equipe. Eventualmente, para projetos específicos, eram chamados colaboradores temporários, especialmente na área de jornalismo.

\section{NOVO OLHAR, NOVO PRODUTO}

Em 1998, o Estúdio Elifas Andreato, tento firmado compromisso com a Editora Globo para a execução de um projeto especial ligado à música brasileira, ampliou sua equipe até assumir sua configuração atual - formada por equipes fixas de pesquisa e produção de conteúdo. Nessa época, também, começou a se delinear o que se tornaria o principal produto da empresa: o Almanaque Brasil de Cultura Po-

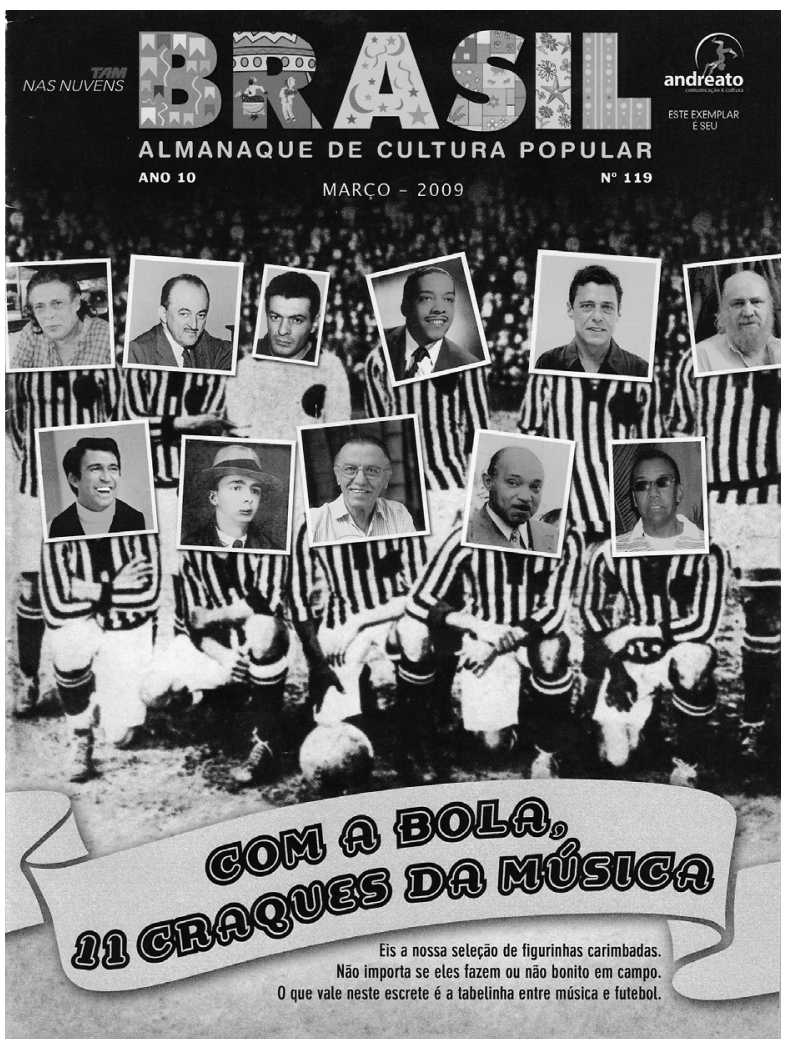

Capa do Almanaque Brasil de Cultura Popular, edição de março de 2009. pular, publicação mensal abordando a história, a cultura e as artes do Brasil, com tiragem de 60 mil exemplares. Sustentado financeiramente por anúncios, é distribuído gratuitamente nos voos da companhia aérea TAM, além de escolas e organizações parceiras.

A empresa continuava participando de outros projetos nas áreas editorial, de ambientação cenográfica e de identidade visual e corporativa. No início de 2005, mudaria seu nome para Andreato Comunicação $e$ Cultura-destacando a empresa não apenas como o local onde o fundador trabalha como artista gráfico, mas também como sede de uma equipe de profissionais aptos a desenvolver projetos de comunicação. 
3. HARVEY, David. Condição pós-moderna. São Paulo: Loyola, 1993.

4. SIQUEIRA, Holgonsi Soares Gonçalves. Estruturas de informação e comunicação. Disponíve em: <http://www.angelfire.com/sk/holgonsi/informa.html >. Acesso em: 03 out. 2005.

5. Ibid. comunicação \& educação • Ano XVII • número 1 • jan/jun 2012

\section{PÓS-MODERNIDADE E AFIRMAÇÃO DE IDENTIDADE}

É possível traçar um paralelo entre a fase de transição vivenciada pela Andreato e a revisão das relações de trabalho e produção contemporâneas ou pós-modernas. A revolução tecnológica, cultural e econômica ocorrida no final do século XX causou mudanças paradigmáticas no modo de se pensar a sociedade e as instituições. Aliados ao fortalecimento do capital volátil como responsável pela importância econômica e política das nações, os novos meios de produção fazem com que o emprego deixe de ser o símbolo do desenvolvimento nacional e do crescimento econômico. Consequentemente, perdem força os movimentos organizados de trabalhadores e surgem novas instituições, regidas pela flexibilização das relações de trabalho.

Pode-se dizer que a pós-modernidade tem início com a passagem das relações industriais de produção para as pós-industriais, baseadas não apenas na troca de mercadorias, mas fundamentalmente em serviços e trocas de bens abstratos, entre eles a informação. Ao influenciar a reestruturação dos processos produtivo e do trabalho, o novo paradigma informacional, guiado, sobretudo, pela velocidade de trânsito da informação, também trouxe profundas mudanças nas concepções de tempo e de espaço ${ }^{3}$. A comunicação em tempo real torna necessárias respostas rápidas a situações que se reconfiguram em alta velocidade, especialmente dentro das atividades econômicas e produtivas. Dessa forma, podemos dizer que o novo paradigma informacional contribui não somente para o surgimento da "sociedade da informação" - considerando que, em maior ou menor grau, a informação foi sempre crucial em todas as sociedades -, mas, sim, de uma "sociedade informacional", que se estrutura em redes e diz respeito a um amplo processo tecnológico, político e sociocultural dentro do qual a informação, mais do que subsídio, se torna também fonte de poder.

Portanto, as oportunidades de crescimento, no novo modelo, dependem do acesso e do lugar ocupado pelos indivíduos no "modo de informação" - ao contrário do que ocorrera na modernidade, em que as oportunidades estavam relacionadas ao lugar no "modo de produção". A partir dessa nova divisão internacional do trabalho, surge uma diversidade de carreiras relacionadas à informação, o que provoca o surgimento de uma nova classe social: a dos "trabalhadores do conhecimento" . No atual modo de operação das empresas, a presença de canais de comunicação integrados, seguros e eficazes é capaz de colaborar grandemente, senão garantir, com a competitividade. Esses canais de comunicação materializam, tanto no aspecto tecnológico como político, a "rede global de interação" necessária à operação da organização na pós-modernidade.

\section{MUDANÇAS NA ORGANIZAÇÃO}

Dentro da Andreato Comunicação e Cultura, apresentou-se, dessa forma, o momento de transição entre a atuação inspirada no trabalho pessoal do artista 
Tradição e mudança • Juliana Winkel

plástico Elifas Andreato e as necessidades empresariais sentidas em um mercado influenciado pela pós-modernidade. Questões como a execução de trabalhos terceirizados ou a forte concorrência do setor de comunicação são influenciadas pela nova corrente econômica e produtiva mundial, ao mesmo tempo que se constituem em desafios organizacionais. A missão da organização foi a de transformar a identidade do fundador, assim como seu trabalho e seu estilo, em marcas institucionais e capital simbólico.

\section{O PROBLEMA}

Levando-se em conta o histórico da Andreato Comunicação e Cultura, percebemos alguns pontos fundamentais a serem considerados no momento de se estudar a comunicação como ferramenta de gestão organizacional. Entre eles estão: a potencialização do uso dos meios de comunicação digital; a valorização da cultura nacional em uma época de globalização; a reafirmação da equipe como um grupo de valores e objetivos comuns, em conformidade com a filosofia do fundador da organização; e a reafirmação da identidade da empresa em um mercado altamente competitivo.

À complexidade da questão, contrapõe-se a vasta história de participação social de Elifas Andreato no cenário cultural brasileiro - histórico, por si só, suficiente para definir o perfil da instituição.

Dessa forma, pôde-se formular o problema-foco da pesquisa da seguinte maneira: Como adequar a comunicação externa da empresa às expectativas do mercado, ao mesmo tempo em que se afirma a imagem institucional da mesma como agência diferenciada na produção de conteúdo cultural brasileiro?

\section{A PESOUISA - METODOLOGIA}

Eis os aspectos que nortearam o projeto de pesquisa:

1) Como uma organização fortemente marcada pela figura e carreira do fundador pode afirmar uma imagem institucional que, sem se opor a ele, o transcende?

2) Como a comunicação pode auxiliar na imagem de uma instituição que é, ao mesmo tempo, potencializadora do trabalho de seu fundador e de seus clientes?

3) Como integrar, em uma diretriz e linguagem de divulgação comuns, produtos tão diversos - desde trabalhos artísticos individuais até periódicos e produção gráfica para clientes?

4) Como atualizar a comunicação e as marcas mais significativas do trabalho da organização, de acordo com os novos padrões de comunicação da pós-modernidade? 
comunicação \& educação • Ano XVII • número 1 • jan/jun 2012

\section{INSTRUMENTOS}

A pesquisa objetivou o posicionamento da marca combinado ao uso de estratégias de maior alcance em comunicação, com destaque para as novas mídias. O público diretamente envolvido foram os clientes da Andreato Comunicação e Cultura e os usuários do site da organização. Sendo uma pesquisa que privilegia a busca de informações detalhadas e, até certo ponto, particulares em cada caso - como a relação empresa-cliente; a ligação feita pelos clientes entre a figura do fundador e o restante da equipe; a identidade dos produtos -, optou-se pelo método qualitativo e não probabilístico de coleta de dados.

Foram realizadas entrevistas com os principais agentes responsáveis pelo perfil da instituição, assim como com clientes de representatividade. Os dados são opinativos e avaliativos, visto que o público pesquisado é especializado, já tendo tido contato com os temas-objeto da pesquisa durante tempo suficiente para balizar, pela experiência, suas impressões.

Havendo a intenção de utilizar as mídias digitais, em especial a Internet, no projeto de intervenção, procedeu-se ainda a uma pesquisa de sites de organizações afins para detectar a forma como trabalharam questões como a relação cliente/empresa, serviços, identidade visual e conteúdo informativo.

\section{ANÁLISE DE DADOS}

Entre as informações recolhidas junto aos integrantes da direção da Andreato, houve unanimidade com relação ao que consideraram o principal desafio da organização: a dicotomia entre a manutenção de sua herança artística e os princípios de trabalho e a adaptação às novas formas de produção e de comunicação.

Outro desafio constatado foi o aumento da divulgação dos trabalhos da empresa como fruto de uma atuação de equipe. A marca Andreato aparece como essencialmente ligada à figura do fundador. Mostrou-se a necessidade de afirmar que o patrimônio cultural construído pela empresa, mais do que o traço de uma carreira individual, é resultado da trajetória de toda uma instituição, compartilhado por um grupo inteiro de profissionais.

Ao mesmo tempo, mostrou-se importante cultivar a presença de Elifas como ainda constante e responsável pelas diretrizes principais da organização, intimamente ligada ao espírito do que ali é produzido - sem que isso tolha a representatividade da equipe de modo geral. Tratou-se, aqui, de valorizar a marca sem que se abra mão do que é forte na construção de sua identidade.

Entre os entrevistados, manifestou-se o desejo de preservação da identidade da organização como empresa que trabalha, primordialmente, com temas ligados à cultura nacional. A preocupação com valores nacionais pode ser entendida, inclusive, como uma tendência da pós-modernidade: enquanto na 
Tradição e mudança • Juliana Winkel

década de 1960 o nacionalismo era utilizado para fins de afirmação nacional interna, atualmente a cultura nacional é ferramenta de afirmação de identidade perante as demais culturas globais - ainda que as identidades nacionais recebam influências externas, tornando elástico o conceito de cultura nacional. Nesse sentido, torna-se fundamental, para a conquista de espaço no mercado, que uma marca se identifique culturalmente com uma parcela considerável da população, investindo em projetos culturais relacionados à região com a qual se busca interagir.

O destaque à cultura brasileira é visto como valor indissociável da identidade da Andreato, ainda que signifique, em alguns momentos, restrição de mercado. No entanto, a especificidade do olhar da empresa sobre os trabalhos é vista como benéfica, pois reafirma seu diferencial.

\section{IMPRESSÕES COLHIDAS DURANTE A PESQUISA: FORTALECIMENTO DA MARCA E NOVAS MÍDIAS}

A pesquisa comprovou que o desafio enfrentado pela organização estava em fortalecer sua marca como instituição geradora de conteúdo específico e, ao mesmo tempo, potencializar a utilização de ferramentas digitais junto a trabalhos que, já possuindo alto nível de apresentação no meio impresso, trazem, em seu perfil, a essência da cultura pós-moderna - ou seja, a versatilidade da abordagem por meio de diversos tipos de linguagem (textos, filmes, canções), assim como em formatos diversos (CDs, DVDs, sites e outros).

Foram sugeridas ainda outras medidas para potencializar a comunicação da instituição, confirmadas por meio da pesquisa de campo:

- Evidenciar, diante do mercado, a continuidade do trabalho do fundador Elifas Andreato como identidade comum a todos os trabalhos produzidos pela empresa, de forma que cada membro da equipe fosse visto como detentor de competências diferenciadas e, ao mesmo tempo, fundamentais para a manutenção dos ideais originais da organização.

- Aproveitar o potencial das mídias digitais, que têm muito a oferecer em termos de mobilidade, dinamicidade e identificação com o conteúdo dos produtos da Andreato Comunicação e Cultura.

- Evidenciar a empresa como referência na produção de conteúdo específico sobre cultura brasileira. Como passo importante nesse sentido, mostrou-se necessário destacar, nos projetos próprios, a imagem da marca Andreato em primeiro plano como responsável pelos produtos, ao invés de evidenciar o nome dos parceiros e apoiadores antes do nome da própria marca.

- Salientar a história de valorização da cultura nacional, por meio da comunicação criativa voltada para os aspectos inusitados, curiosos e autênticos da história e da cultura brasileiras - traços expressos como diferenciais no mercado. 
comunicação \& educação • Ano XVII • número 1 • jan/jun 2012

\section{O PROJETO}

As propostas aqui descritas, elaboradas com base na pesquisa de campo e no estudo das possibilidades de comunicação da empresa, fazem parte de um amplo plano de afirmação da marca Andreato junto a seu público direto, levando em conta o cenário de comunicação e produção cultural contemporâneo e contribuindo para ampliar a visibilidade da marca, aumentando também o aproveitamento das novas mídias dentro de sua comunicação:

- Destacar a existência, na empresa, de uma equipe polivalente, em sintonia com o trabalho do fundador. Para reforçar a linha de atuação da organização como algo único, guiado pelos preceitos de trabalho e criação de seu fundador, é interessante mostrar que esses princípios estão presentes no cotidiano de todo o grupo, evidenciando a existência de um sistema ágil, com equipes responsáveis por cada etapa do trabalho e gerenciadas por especialistas em suas áreas. Para isso, fez-se necessário mostrar de forma mais efetiva, nos canais de comunicação da empresa, o perfil desses profissionais.

- Aumentar os canais de divulgação da organização junto aos clientes. Além dos canais de comunicação digitais, sugeriu-se a elaboração de materiais específicos direcionados a potenciais clientes, como portfólios e folders contendo uma apresentação dos principais projetos da organização, de seu perfil e de seus diferenciais. Um serviço de assessoria de imprensa, que divulgue os trabalhos de Elifas e da organização junto a portais de comunicação e à mídia especializada, também traria grande impulso ao nome da marca.

- Destacar a marca da empresa em relação às marcas de parceiros e patrocinadores. Diferentemente de publicações corporativas, tanto o Almanaque Brasil quanto sua versão digital são publicações de interesse geral, cuja linha editorial é de responsabilidade da própria Andreato - embora receba apoio de outras instituições. Nesse sentido, é interessante destacar a logomarca da organização sempre em primeiro lugar, sendo seguida pelas logomarcas dos apoiadores.

- Reformular a comunicação digital da empresa. A comunicação digital da organização, centrada em dois sites independentes - o site da própria organização e o site do Almanaque Brasil -, pode ser ampliada e reformulada, seguindo padrões identificados com o espírito criativo presente nas publicações impressas.

\section{CONSIDERAÇÕES FINAIS}

O novo sistema de trabalho e produção, advindo da revolução tecnológica e estética da pós-modernidade, nos insere em processos sociais em que a informação adquire papel central. A informatização dos meios de comunicação gera, em cada profissional, a urgência da atualização. Não nos podemos esquecer, 
Tradição e mudança • Juliana Winkel

no entanto, de que a tecnologia só será plenamente aproveitada em todas as suas potencialidades se estiver inserida na realidade de cada organização ou comunidade, afinada com sua história, suas necessidades e expectativas. $\mathrm{Na}$ análise desses cenários e no estabelecimento de vias de operação eficazes para a tecnologia informacional, o Gestor de Comunicação tem papel fundamental.

É essencial aos profissionais de comunicação adquirir competências necessárias não só ao gerenciamento da comunicação, mas, também, à capacidade de estabelecer correlações eficazes dentro dos diversos canais comunicacionais. $\mathrm{O}$ processo de produção contínuo e periódico, baseado em modelos predeterminados, característico da modernidade, agora está aliado também ao conhecimento intensivo e à flexibilidade da mensagem, fundados no planejamento e na elaboração de projetos que se adaptem, especificamente, a cada trabalho. É preciso ter em vista, de forma permanente, a inter-relação entre o próprio trabalho e o contexto vasto e desafiador em que a comunicação está inserida dentro de uma organização, assim como dentro da realidade do público envolvido em suas diversas etapas. Mais do que simplesmente atuar em comunicação, é necessário refletir sobre suas práticas, conferindo-lhes autenticidade, potencializando seus resultados, extraindo delas o melhor de suas capacidades em seu ambiente.

Dessa forma, a comunicação deixa de atuar em caráter acessório, como complemento às demais atividades de gestão, para assumir verdadeiro papel centralizador de processos, fortificador de atitudes e formador da cultura do espaço em que está inserida - seja ele uma empresa, uma comunidade, seja o público crescente e diversificado que a ela tem acesso pela Internet. Em todos os casos, em maior ou menor escala, o comunicador será sempre um mediador das demandas não só comunicacionais, mas também sociais e culturais vindas da comunidade em que atua ${ }^{6}$.

Numa época de transformação social acelerada e constante, os comunicadores assumem papel decisivo na evolução do espaço em que atuam. Ao gestor da comunicação, cabe aproveitar o imenso potencial agregador dos meios de comunicação contemporâneos para, em vez de separar, integrar, transformando a experiência coletiva não num espaço transitório, mas sim de encontro.

\section{REFERÊNCIAS BIBLIOGRÁFICAS}

FALCÃO, Maria do Carmo. Cotidiano: conhecimento e crítica. São Paulo: Cortez, 1989.

HALL, Stuart. A identidade cultural na pós-modernidade. Rio de Janeiro: DP\&A Editora, 1999.

HARVEY, David. Condição pós-moderna. São Paulo: Loyola, 1993.

LOPES, Maria Immacolata V. Sobre um novo projeto pedagógico no campo da Comunicação. In: BACCEGA, Maria Aparecida (org.). Comunicação e cultura: um novo profissional. São Paulo: CCA/ECA/USP, 2000.
6. LOPES, Maria Immacolata V. Sobre um novo projeto pedagógico no campo da Comunicação. In: BACCEGA, Maria Aparecida (org.) Comunicação e cultura: um novo profissional. São Paulo: CCA/ECA/USP, 2000. p. 16. 
comunicação \& educação • Ano XVII • número 1 • jan/jun 2012

\section{Endereços eletrônicos}

ARBEX JR, José. O Terceiro Mundo - Américas. s/l, s/d. Disponível em: <http:/ / www.tvcultura.com.br/aloescola/historia/guerrafria/guerra11/terceiromundoamericas.htm>. Consulta em: 16 ago. 2005.

SIQUEIRA, Holgonsi Soares Gonçalves. Estruturas de informação e comunicação. Disponível em: <http://www.angelfire.com/sk/holgonsi/informa.html $>$. Acesso em 03 out. 2005.

. Novo paradigma informacional. Disponível em: $<\mathrm{http}: / /$ www.angelfire. com/sk/holgonsi/informacional.html>. Acesso em: 03 out. 2005. 